\title{
Task Co-Representation in Aging: An Event-Related Potential Study
}

\author{
Kimiko Kato*, Kazuhito Yoshizaki \\ Department of Psychology, Aichi Shukutoku University, Nagakute, Japan \\ Email: *kimi@asu.aasa.ac.jp
}

How to cite this paper: Kato, K. and Yoshizaki, K. (2020) Task Co-Representation in Aging: An Event-Related Potential Study. Journal of Behavioral and Brain Science, 10, 455-469. https://doi.org/10.4236/jbbs.2020.1010029

Received: September 22, 2020

Accepted: October 27, 2020

Published: October 30, 2020

Copyright $\odot 2020$ by author(s) and Scientific Research Publishing Inc. This work is licensed under the Creative Commons Attribution International License (CC BY 4.0).

http://creativecommons.org/licenses/by/4.0/

(c) (i) Open Access

\begin{abstract}
The goal of the present study was to investigate age-related changes in attentional allocation for shared task representations during joint performance; event-related potentials were recorded while participants performed a modified visual three-stimulus oddball task, both alone and together with another participant. Younger adults and older adults (14 each) participated in the study. Participants were required to identify rare target stimuli while ignoring frequent standards, as well as rare non-targets assigned to a partner's action (i.e., no-go stimuli for one's own task). ERP component, nogo-P3 and P3b were measured to investigate the inhibition and the attentional allocation to the partner's stimuli. Results showed that younger adults elicited larger frontal no-go P3 and parietal P3b for non-targets in the joint than in the individual condition. Contrary to expectation, older adults induced frontal no-go P3 in the joint condition not in the individual condition. In the sharing of the task with another, the result suggested that the efficiency of matching of incoming information with the representation of the other's task declined with age, whereas aging did not affect the suppression of incorrect preparation of motor responses instigated by this representation.
\end{abstract}

\section{Keywords}

Aging, Joint Action, Shared Task Representation, Event-Related Potential

\section{Introduction}

According to the frontal aging hypothesis of West (1996), cognitive functioning first begins to decline with age in the prefrontal brain regions [1]. Cognitive decline in older adults has been demonstrated in terms of attentional functions, such as focusing on task-relevant stimuli, inhibiting irrelevant information, and dividing attention between multiple ongoing tasks [2]. Electrophysiological stu- 
dies [2] have suggested that changes in prefrontal cortex function in aging are related to impaired sustained attention and lack of suppression of distractors. Age-related decline of divided attention can be seen in everyday life as well as in structured laboratory settings; for example, during driving while talking to a fellow passenger and cooking while managing family life [3] [4]. This age-related decline in attentional function appears to impair the ability to pay attention to, and acquire, information necessary for maintaining good social relations in daily life.

Actions and decisions accomplished by means of interactions between individuals vary with context and circumstances. This crucial ability has been attributed to a common coding system that links perceived events to intended actions and activates the same representations or common codes [5]. Neural evidence for common coding has been identified in mirror neurons in the premotor cortex of the macaque monkey [6] [7]. Common codes enable us to infer another's intention from observed actions, activating representations in our own action system. Shared representations with partners have been demonstrated using a joint action paradigm in a number of behavioral studies [8]-[13] as well as event-related potential (ERP) studies [14] [15] [16] [17] [18].

Typically, the joint action paradigm provides two conditions: a joint and an individual condition. A joint condition requires two partners sitting next to each other to respond to different types of information with the same fast response task embedded in it. In an individual condition, a participant, alone, is asked to respond only to the specified stimulus information, not to the other, i.e., go-nogo task. In our previous study [15], ERP activity was recorded while participants performed an auditory three-stimulus oddball task alone (individual condition) and with another participant (joint condition). In this paradigm, participants were required to identify frequent standard tones and rare target tones using designated keys, while ignoring rare non-targets assigned to a partner's action (i.e., no-go stimuli for the participant's own task performance). This research focused on the P3 component of ERP in a go-nogo task. In the go-nogo task, two types of P3 are observed [19]. For no-go trials, the no-go P3 is evoked in frontal and central sites, whereas go trials elicit P3b in central-parietal maximum. Parietal P3b has been measured as a "culmination of multiple cognitive processes" [20], such as attentional allocation [21] [22] [23], context updating [22] [24], and the timing of stimulus classification [25] [26], while frontal no-go P3 has been found with response inhibition or suppression of action tendencies [16] [17] [18]. Kato et al. (2016) observed P3b for targets in both individual and joint conditions, whereas $\mathrm{P} 3 \mathrm{~b}$ for non-targets was elicited only in the joint condition [15]. P3b for non-targets was interpreted as a reflection of intentional stimulus classification for non-targets, based on the notion of co-representations of one's own and another's actions formed in a joint setting. In addition, the emergence of P3b and no-go P3 for non-targets in the joint condition was delayed relative to $\mathrm{P} 3 \mathrm{~b}$ for targets, suggesting that shared task representations are 
serially applied to stimulus processing and that one's own representations precede those of another individual.

The present experiment was designed to examine age-related changes in attentional allocation for shared task representations during joint performance. A three-stimulus oddball paradigm was combined with a cyberball situation, in which participants played a ball-tossing game on a computer [27]. This combined paradigm was used to emphasize collaborative behavior in a joint action situation and to keep participants interested in performing the experimental task. ERP activity was recorded from each participant who performed the task alone beside an empty chair (individual condition) and from paired participants who sat side by side (joint condition). In these social contexts, three classes of stimuli (standard, target, and non-target) were presented on a computer monitor, which initially displayed four squares (boxes) in a square arrangement. A ball (black circle), the standard, was presented frequently and alternately in the upper left and right boxes, as if it were tossed between those boxes. For left-seated participants, the balls delivered to the lower left and right boxes were assigned as the target and non-target, respectively, and participants were required to press a left button for targets (i.e., go trials) and withhold button-pressing for non-targets and standards (i.e., no-go trials). For right-seated participants, the assignment of stimuli (targets and non-targets) and response-button position were reversed. ERP indices included frontal no-go P3 and parietal P3b.

We hypothesized that ERP differences in older adults would differ from those in younger adults. Specifically, we predicted that cognitive decline in older adults would make it difficult for them to allocate attentional resources appropriately for representation of the other's task in a joint context. This prediction would be revealed by non-target P3b. For younger adults, non-target P3b should be larger in the joint condition than in the individual condition, because younger adults, who have abundant attentional resources, can readily form a representation of the other's task and apply it to stimulus matching, as seen in the previous study [15]. On the other hand, for the above reasons, older adults, for whom attentional resources are less abundant relative to younger adults, should not show any differences between social contexts in terms of non-target P3b. In addition, we predicted that the processing of misleading stimuli would elicit frontal no-go P3, which reflects incorrect preparation and subsequent suppression of motor responses, for the participant's own task-irrelevant non-targets. On the other hand, for older adults, difficulties in forming and applying a partner's task representations should result in attenuation of no-go P3 measures for non-targets in the joint condition. P3b for targets should not differ between social contexts, because processing of stimulus classifications is executed based on one's own representations. In the individual condition, both younger and older participants should show a large P3b for targets, as in a three-stimulus oddball task [28] [29]. There should be no difference in P3b for frequently occurring standard stimuli 
between the joint and individual conditions, as in the previous study [15].

\section{Method}

\subsection{Participants}

Fourteen younger adults (age range: 20 - 30 years, $M=21.8, S D=2.8,1$ male and 13 females) and fourteen older adults (age range: $68-74$ years, $M=70.9$, $S D=1.7,8$ males and 6 females) participated. The number of participants was determined by a previous study that investigated attentional control in aging using a three-stimulus oddball task [30]. Older adults were recruited from a community silver human resource center. There was no difference in years of education between the two age groups (younger adults, $M=15.1$ years, $S D=2.3$; older adults, $M=13.7$ years, $S D=2.0 ; t(26)=1.63, p=0.11)$. All participants had normal or corrected-to-normal vision. The Mini Mental State Examination (MMSE) [31], a quick and easy measure of cognitive function, was administered to the older adults. All older participants were cognitively intact (MMSE score range: $28-30, M=29.5, S D=0.7)$. However, the data of three older adult participants were excluded because of excessive artifacts, such as eye blinks.

All participants gave their informed consent to take part in this experiment and received a modest monetary remuneration. All procedures were approved by the Ethical Committee of Aichi Shukutoku University (No. 2016-04-r01), the date of the approval was June 27, 2017.

Each pair of participants in the joint condition was acquaintances. Younger adults were selected from the same class in the same university department. Older adults were members of the same community silver human resource center, who had participated several times in the authors' research and had previously met each other.

\subsection{Stimuli}

As shown in Figure 1, a computer monitor initially displayed four squares (boxes) in a square arrangement. All four squares were presented continuously during the block. There were three stimulus classes: standard, target, and non-target. The standard (appearance probability $(\mathrm{p}=0.6)$ was a black circle (ball) presented either to the upper left $(p=0.3)$ or right box $(p=0.3)$, whereas the target and non-target ( $\mathrm{p}=0.2$ for each) were delivered either to the lower left $(\mathrm{p}=0.1)$ or right box $(\mathrm{p}=0.1)$. The standard, target, and non-target stimuli were presented in a random order in one of the squares. For left-seated participants, the target was a ball that appeared in the lower left box, whereas the non-target was a ball that appeared in the lower right box. The assignment of target and non-target was reversed for right-seated participants.

The fixation point at the center of the screen was a black plus sign, $0.7^{\circ} \times 0.7^{\circ}$ of visual angle in width and height. Four boxes were drawn in black outline, and each was $3.8^{\circ}$ square in size. Vertical and horizontal distances from the fixation point to each box were $3.7^{\circ}$ and $4.1^{\circ}$, respectively. The black circle (ball) was $2.3^{\circ}$ 


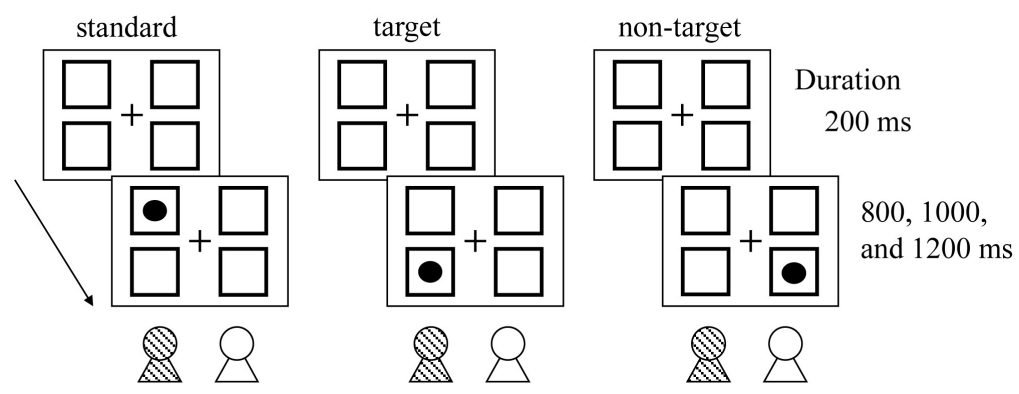

Figure 1. Illustration of the experimental setup. The three stimulus classes (standard, target and non-target) for left-seated participants are shown.

in diameter. All stimuli were displayed on a white background.

\subsection{Procedure}

The experiment was conducted in a quiet room. In the joint condition, paired participants sat side by side in front of a computer monitor, and ERPs were recorded from both participants. In the individual condition, each participant sat next to an empty chair. Viewing distance was $70 \mathrm{~cm}$ from the monitor. In both social contexts, participants were told to play a ball-tossing game. They were instructed to gaze at a fixation point and to minimize blinking while performing the task. Each trial began with the presentation of the four boxes and the fixation point for $200 \mathrm{~ms}$, and then a ball was presented in one of the four boxes for a random duration of 800, 1000, or $1200 \mathrm{~ms}$ (see Figure 1). Left-seated participants were required to press a response button with their left index finger for targets (balls appearing in the lower left box) as quickly and accurately as possible and to ignore non-targets and standards. Right-seated participants used their right index finger for button pressing. Response times were recorded from the onset of the target to the participant's response using a Response Pad (Cedrus Company, San Pedro, CA, USA). The next trial began immediately. Stimuli were displayed using SuperLab 4.5 (Cedrus Company, San Pedro, CA, USA).

Each participant completed ten blocks of 60 trials in each social context. Half of the participants in each age group began with the individual condition, and half began with the joint condition. The side on which the participant sat in the chair changed every five blocks within each context. The order of administration across the social context and seated chair conditions (associated with target position and response finger) was counterbalanced across participants in each age group.

\subsection{Electrophysiological Recording and Data Analysis}

EEG activity was recorded from the Fz, Cz, Pz, C3, C4 (according to the International 10/20 system), and right earlobe sites using $\mathrm{Ag}-\mathrm{AgCl}$ electrodes. Vertical EOG was recorded from Fpz. These recording sites were referenced to the left earlobe. EEG and EOG signals were amplified with a band-pass of $0.05-30 \mathrm{~Hz}$, and data were collected at a sampling rate of $1000 \mathrm{~Hz}$. All electrode impedances 
were maintained below $5 \mathrm{k} \Omega$. EEG data were re-referenced off-line to the average of the left and right earlobes. ERPs were averaged separately for each electrode site in each of the 12 experimental categories of three stimulus classes (standard, target, and non-target) by social context (individual and joint) and age group (younger and older). The averaging epoch began $100 \mathrm{~ms}$ before stimulus onset and lasted for $1000 \mathrm{~ms}$. Averaged ERPs were aligned to the $100 \mathrm{~ms}$ pre-stimulus baseline. Trials containing EOG artifacts (greater than $\pm 100 \mu \mathrm{v}$ ) were excluded. The trials excluded from analyses due to artifacts were $21.4 \%$ and $33.8 \%$ of all trials in younger and older adults, respectively. On average, in younger adults, the respective numbers of trials comprising ERP waveforms for standard, target, and non-target stimuli were 263,95 , and 87 in the individual condition, and 286, 103, and 98 in the joint condition. In older adults, the corresponding numbers of trials comprising ERP waveforms were 203, 89, and 72 in the individual condition, and 214, 90, and 86 in the joint condition. ERP processing was performed by the MaP1200 (Nihonsanteku Company, Osaka, Japan).

ERP data were quantified by measuring mean amplitudes at $\mathrm{Fz}, \mathrm{Cz}$, and $\mathrm{Pz}$. Behavioral data were quantified by counting as hits button presses that occurred within a $100-1200 \mathrm{~ms}$ time window after target onset. Responses for non-target and standard stimuli were counted as false alarms. Statistical analyses of ERP and behavioral measures were performed using repeated measures ANOVAs. Results were corrected with Huynh-Feldt $\varepsilon$ corrections. The Holm method was used for post-hoc multiple comparisons, with a significance level of 0.05 .

\section{Results}

\subsection{Performance}

Performance data are summarized in Table 1. Two-way ANOVAs with the factors of age group (young and old) and social context (individual and joint) were conducted on the behavioral measures of reaction times to targets and false alarms to standards and non-targets. However, no significant main effects or interactions were found for any measures (RT: $F \mathrm{~s}(1,23)<1.92, p \mathrm{~s}>0.179, \eta_{p}^{2}<$ 0.08; false alarms to standards: $F_{\mathrm{s}}(1,23)<1.99, p \mathrm{~s}>0.172, \eta_{p}^{2}<0.08$; false alarms to non-targets: $\left.F_{\mathrm{S}}(1,23)<2.43, p s>0.133, \eta_{p}^{2}<0.10\right)$.

\subsection{ERPs}

Figure 2 shows grand averaged ERP waveforms at $\mathrm{Fz}, \mathrm{Cz}$, and $\mathrm{Pz}$ for the individual and joint conditions. A prominent effect of joint action can be seen for non-target (i.e., partner's target) stimuli. A positive deflection peaking around $500 \mathrm{~ms}$ post-stimulus emerged at the three electrode sites in the joint condition, whereas ERPs in the individual condition produced no visible positivity between 400 and $700 \mathrm{~ms}$ post-stimulus. This joint action effect for non-targets appears to be reduced in older adults. For standard and target stimuli, ERPs indicate no differences, such as those observed for non-targets in both groups. Irrespective 
Table 1. Mean reaction times (ms), hit rates (\%) to targets, and false alarm rates (\%) to standards and non-targets in individual and joint conditions for younger and older adults.

\begin{tabular}{ccccc}
\hline & \multicolumn{2}{c}{ Younger } & \multicolumn{2}{c}{ Older } \\
\cline { 2 - 5 } & Individual & Joint & Individual & Joint \\
\hline Reaction time & 420 & 400 & 440 & 419 \\
Target hits & 98.8 & 99.0 & 99.0 & 98.5 \\
False alarms & & & & \\
Standard & 0.26 & 0.20 & 0.45 & 0.61 \\
Nontarget & 1.55 & 0.71 & 1.36 & 1.06 \\
\hline
\end{tabular}

of age group and social context, a typical, parietally-dominant P3b peaking around $400 \mathrm{~ms}$ was observed for targets.

The ERP components to be analyzed were selected on the basis of visual inspection of grand averages. P3b, which is a notable component for an oddball task, was determined as the maximal amplitude between 200 and 500 ms post stimulus. In addition, we quantified mean amplitudes in the $400-700 \mathrm{~ms}$ time window in which the difference between the social contexts for non-targets was visible in both age groups. First (3.2.1), the positive amplitudes for $200-500 \mathrm{~ms}$ in younger adults and older adults were analyzed for the individual conditions to confirm the robust effects reported by earlier studies that used a three-stimulus oddball task (Katayama \& Polich, 1996, 1999). Second (3.2.2 and 3.2.3), for each stimulus condition within these two time windows (200 - $500 \mathrm{~ms}$ and $400-700$ $\mathrm{ms}$ ), a three-way ANOVA was conducted, with a between factor of age group (younger and older) and within factors of electrode site ( $\mathrm{Fz}, \mathrm{Cz}$, and $\mathrm{Pz}$ ), and social context (individual and joint).

\subsubsection{Age Group $\times$ Stimulus Condition $\times$ Electrodesite}

Significant effects included the main effect of electrode site, $F(2,46)=30.68, p<$ $0.001, \eta_{p}^{2}=0.57, \varepsilon=0.929$, and the two-way interactions of age group $\times$ electrode site, $F(2,46)=12.04, p<0.001, \eta_{p}^{2}=0.34$, and stimulus $\times$ electrode site, $F(4,92)=9.29, p<0.001, \eta_{p}^{2}=0.29, \varepsilon=0.484$. The three way interaction was also significant, $F(4,92)=8.55, p=0.001, \eta_{p}^{2}=0.27$. Further analyses revealed that for younger adults, targets elicited the largest $\mathrm{P} 3 \mathrm{~b}$ relative to other stimuli only at $\mathrm{Pz}, F(2,138)=15.00, p<0.001, \eta_{p}^{2}=0.59$, and not at $\mathrm{Fz}, F(2,138)=$ $0.05, p=0.95, \eta_{p}^{2}=0.00$, or $\mathrm{Cz}, F(2,138)=2.76, p=0.067, \eta_{p}^{2}=0.21$, with no difference in $\mathrm{P} 3 \mathrm{~b}$ between standards and non-targets. For older adults, the three stimuli did not differ in P3b at any electrode sites, $F \mathrm{~s}(2,138)=0.15-1.09$, $p s>0.34, \eta_{p}^{2}=0.01-0.09$.

\subsubsection{0 - $500 \mathrm{~ms}$ Time Window}

For standards in the 200 - 500 ms time window, a significant main effect of electrode site was found, $F(2,46)=9.89, p=0.001, \eta_{p}^{2}=0.30, \varepsilon=0.812$, suggesting that the positive deflection increased at the centro-parietal site. 


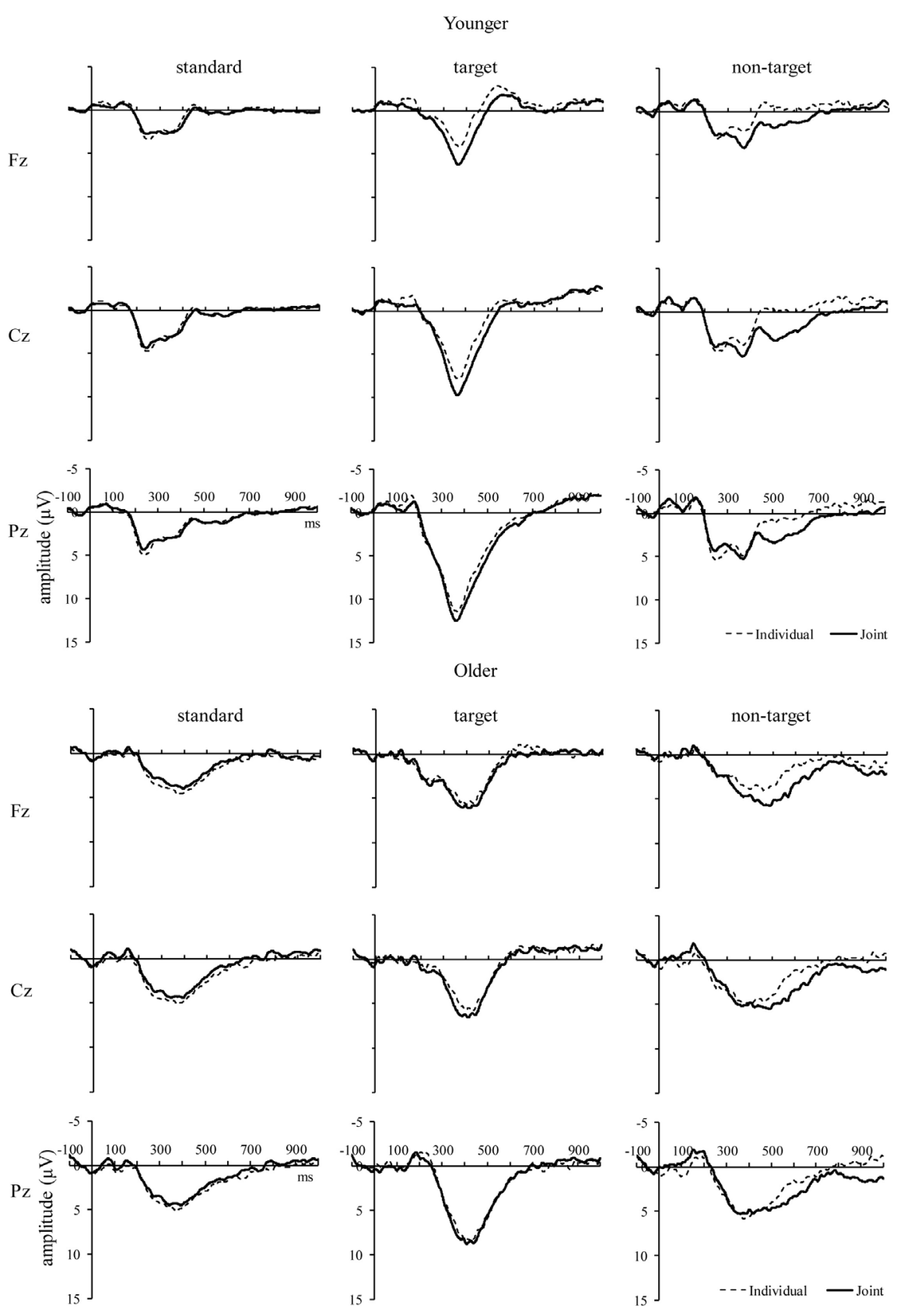

Figure 2. Grand averaged ERPs at Fz, Cz, and Pz. Waveforms are superimposed for individual (dotted lines) and joint (solid lines) conditions and shown separately for standard, target, and non-target stimuli. For each stimulus class, waveforms are shown separately for younger (upper panel) and older adults (lower panel). Negativity is represented by the up direction.

For targets, there were a significant main effect of electrode site, $F(2,46)=$ 22.34, $p<0.001, \eta_{p}^{2}=0.49, \varepsilon=0.914$, and a significant interaction of age group $\times$ electrode site, $F(2,46)=12.22, p<0.001, \eta_{p}^{2}=0.35, \varepsilon=0.791$. Further analyses showed $\mathrm{Pz}$ maximal positivity in the younger adults, $F(2,46)=32.59, p<0.001$, $\eta_{p}^{2}=0.59$; on the other hand, older adults did not show any differences in amplitude between electrode sites $F(2,46)=1.97, p=0.15, \eta_{p}^{2}=0.08$. In addition, 
the main effect of social context was significant, $F(1,23)=4.91, p=0.036, \eta_{p}^{2}=$ 0.18 , suggesting that enhancement of positivity was larger in the joint condition than in the individual condition.

For non-targets, a significant main effect of electrode site, $F(2,46)=11.99, p<$ $0.001, \eta_{p}^{2}=0.34, \varepsilon=0.850$, and a significant interaction of age group $\times$ electrode site, $F(2,46)=3.89, p<0.027, \eta_{p}^{2}=0.14$, were found. Further analyses revealed that positivity increased from central site to parietal site for younger adults, $F(2,46)=13.94, p<0.001, \eta_{p}^{2}=0.38$, whereas there were no amplitude differences between electrode sites for older adults, $F(2,46)=1.93, p=0.16, \eta_{p}^{2}=$ 0.08 . In addition, a significant interaction of electrode site $\times$ social context was found, $F(2,46)=11.35, p<0.001, \eta_{p}^{2}=0.33, \varepsilon=0.942$. Further analyses revealed that the amplitude of $\mathrm{Fz}$ positivity was greater in the joint condition than in the individual condition, $F(1,69)=5.22, p=0.025, \eta_{p}^{2}=0.69$. This difference was not found at the centro-parietal site, $F \mathrm{~s}(2,46)=0.01-1.50, p s>0.23$, $\eta_{p}^{2}=0.01-0.39$. The positive deflections observed at the centro-parietal and frontal sites in this time window (200 - $500 \mathrm{~ms}$ ) may be regarded as a P3b and a no-go P3, respectively. Other analyses did not show any significant effects ( $p$ s > $0.09)$.

\subsubsection{0 - 700 ms Time Window}

In the $400-700 \mathrm{~ms}$ time window, a significant main effect of age group was found for standards, $F(1,23)=5.11, p=0.034, \eta_{p}^{2}=0.18$, with the positive deflection larger for older adults than for younger adults. A significant main effect of electrode site, $F(2,46)=5.30, p=0.016, \eta_{p}^{2}=0.19, \varepsilon=0.761$, indicated that positivity was greatest at $\mathrm{Pz}$ and least at $\mathrm{Fz}$.

For targets, a significant main effect of electrode site, $F(2,46)=22.01, p<$ $0.001, \eta_{p}^{2}=0.49, \varepsilon=0.899$, suggested that positivity increased from frontal site to parietal site. Furthermore, a significant main effect of social context, $F(1,23)=$ $4.33, p=0.049, \eta_{p}^{2}=0.16$, indicated that positivity was larger in the joint condition than in the individual condition.

For non-targets, significant effects included main effects of age group, $F(1,23)=$ $10.45, p=0.004, \eta_{p}^{2}=0.31$, and social context, $F(1,23)=13.10, p=0.001, \eta_{p}^{2}=$ 0.36 , and a three-way interaction of age group, electrode site, and social context, $F(2,46)=3.37, p=0.047, \eta_{p}^{2}=0.13$. Follow-up analyses of this interaction revealed that for younger adults, positivity was larger in the joint condition than in the individual condition at $\mathrm{Fz}, \mathrm{Cz}$, and $\mathrm{Pz}, F_{\mathrm{s}}(1,69)=5.46-10.98, p \mathrm{~s}<0.022$, $\eta_{p}^{2}=0.45-0.62$. Although a similar pattern was obtained for older adults at $\mathrm{Fz}$ and $\mathrm{Cz}, F(1,69)=8.35$ and 5.11, $p s<0.005$ and $0.027, \eta_{p}^{2}=0.56$ and 0.44 , respectively, no difference between the social contexts were obtained at the parietal site, $F(1,69)=1.64, p=0.204, \eta_{p}^{2}=0.20$. Other analyses revealed no significant differences $(p>0.06)$.

\section{Discussion}

The purpose of the research was to examine the effect of aging on the formation 
of other's task representation in joint action. In this experiment, ERPs were recorded from younger and older adults performing a modified visual three-stimulus oddball task while alone (individual condition) and together with another participant (joint condition). Our previous study of younger adults [15] suggested that co-actors share task representations during stimulus matching. The purpose of the present study was to investigate whether cognitive decline in older adults makes it difficult for them to allocate attentional resources appropriately for representation of another's task.

First, for younger participants in the individual condition, our oddball procedure using visual stimuli replicated the robust findings of a large P3b for targets in a three-stimulus oddball task [28] [29]. By contrast, this large P3b for targets was not observed for older adults. This difference between younger and older adults may have emerged because older adults were less able to classify incoming stimuli unambiguously. According to the P300 context-updating model [32], as a stimulus enters the processing system, it is compared to the previous stimulus, and a sameness judgment is made. An enhancement of P300 amplitude is observed when the incoming stimulus is not the same as the previous stimulus, because participants must freshly allocate attentional resources to the incoming stimulus. To compare previous and current events, participants must maintain the representation in working memory; this is more difficult for older adults. Although our results for older adults may reflect an age-related change in maintenance in working memory, the present data are inadequate to support conclusions about characteristics of older adults with respect to the representation of stimuli in working memory. Therefore, further studies are needed to investigate this age-related change.

The appearance of P3b for frequently occurring standard stimuli, observed predominantly at the parietal site, did not reveal any differences between the social contexts for either age group, consistent with our previous findings [15]. In addition, the positive deflection was larger for older adults than for younger adults in the $400-700 \mathrm{~ms}$ time window. These results suggest that the convergence of activities involved in stimulus evaluation was prolonged in the older adults.

The present study suggests that the presence of others did not affect the processing of targets, which were stimuli important to the participants themselves. As described above, for both age groups there were no differences between social contexts in P3b for targets. Thus older adults as well as young adults were able to allocate attentional resources to important events according to their own task representations.

Enhanced frontal positivity (no-go P3) for non-targets in joint-action settings was observed in the $200-500 \mathrm{~ms}$ time window for older as well as younger adults. This is inconsistent with our prediction. The no-go P3, which reflects incorrect preparation, and then suppression, of motor responses, implies that older participants formed a representation of the partner's task, resulting in im- 
paired processing of the participant's own task-irrelevant non-targets. Interestingly, for older adults, a front-central dominant no-go P3 for non-targets explicitly appeared in the $400-700 \mathrm{~ms}$ time window in the joint condition. This suggests that the inhibition of the partner's stimuli lasted longer for older adults than for younger adults.

The present results support previous findings [15] on the processing of other people's stimuli in young people. Kato et al. (2016) suggested that in situations in which a person is sharing tasks with others (i.e., the joint condition of the present study), two types of processing are being performed: matching incoming information with one's own task representation and matching it with the partner's task representation [15]. As can be seen in Figure 2, the non-target waveform of the younger adults is divided into first and second halves. Specifically, after the positive deflection returns toward baseline, the component once again shifts in a positive direction, a pattern mainly seen in the joint condition. According to Kato et al. (2016), the first and subsequent component correspond respectively to matching to the stimulus that the participant is in charge of and matching to the stimulus that the partner is in charge of [15]. Notably, the results for non-targets in the older adults were distinctly different from those in the younger adults. As can be seen in Figure 2, for older adults, one large positive deflection appeared from $200 \mathrm{~ms}$ to $700 \mathrm{~ms}$ in the non-target waveform. Importantly, this deflection is regarded as a no-go P3, because it was dominant in the front-central area.

Note that the response decision can be made only by matching the incoming stimuli with one's own task representation in working memory. However, the present study demonstrated that the situation of task sharing (i.e., the joint condition) triggered matching to the partner's stimulus (i.e., non-targets) only for younger adults. The deterioration of attentional resources with aging likely led to the age-related difference in non-target processing. Participants should react to their own targets based on their own task representation. Since younger adults have sufficient attentional resources, they can afford to care about the other's action. Consequently, younger adults could allocate resources not only to their own target but also to their partner's target (their non-target). On the other hand, older adults do not execute the matching process to their partner's stimuli. In spite of that, older adults seemed able to inhibit their partner's stimuli. This may reflect efficient use of limited resources by older people.

The disappearance of $\mathrm{P} 3 \mathrm{~b}$ for non-targets in joint settings may reflect the avoidance of errors by older adults. In other words, older adults do not allocate attention for further processing of irrelevant information to own task, irrespective of individual/social situations. Older adults who performed as well as the younger adults showed brain activity more extensive than that of younger adults, while older adults who performed more poorly produced brain activity at the same level as younger adults [33]. That is, processing stimuli in a mode similar to that of younger adults appears to result in a performance decline in older 
adults. Previous research has investigated whether older adults allocate more attentional resources to maintain their cognitive performance than do younger adults; this resource allocation peaks between the late 60 s and early 70 s for cognitively high performers [34]. Older adults in this age range are those who participated in the present research. The present findings of no age differences in $\mathrm{P} 3 \mathrm{~b}$ for targets may reflect this same attentional resource allocation that leads to the maintenance of task performance.

The limitation of this study is that we did not measure cognitive abilities such as working memory. There would be possibility that the age-related differences and non-differences obtain in the present study may depend on the individual differences of cognitive ability.

\section{Conclusion}

In summary, these ERP comparisons of younger and older adults performing a modified visual three-stimulus oddball task alone and together with another participant demonstrate characteristic usage of attentional resources with aging in social contexts. In agreement with our previous study, the younger adults could not only match incoming information with the other person's task representation but also inhibit incorrect preparation of motor responses instigated by representation of the other's task. Although older adults with cognitive decline could not match incoming information with the other's task representation, they could inhibit incorrect preparation of motor responses to non-targets in the social context. Taken together, although, other persons' task representation was formed irrespective of age in a joint setting, it is suggested that aging affects the way of processing based on the representation.

\section{Acknowledgements}

The authors also thank Tsunetaka Okita, $\mathrm{PhD}$, for helpful comments on this article.

\section{Funding}

The research was supported in part by a program grant from the Japan Society for the Promotion of Science KAKENHI Grant Number JP16K04436 to Kimiko Kato.

\section{Approved Information}

The study was approved by the Institutional Ethics Committee of Aichi Shukutoku University (No. 2016-04-r01) consistent with the Declaration of Helsinki of 2008.

\section{Competing Interests}

The authors have declared that no competing interests exist. 


\section{References}

[1] West, R.L. (1996) An Application of Prefrontal Cortex Function Theory to Cognitive Aging. Psychological Bulletin, 120, 272-292. https://doi.org/10.1037/0033-2909.120.2.272

[2] Chao, L.L. and Knight, R.T. (1997) Prefrontal Deficits in Attention and Inhibitory Control with Aging. Cerebral Cortex, 7, 63-69. https://doi.org/10.1093/cercor/7.1.63

[3] Tun, P.A. and Wingfield, A. (1995) Does Dividing Attention Become Harder with Age? Findings from the Divided Attention Questionnaire. Aging, Neuropsychology, and Cognition, 2, 39-66. https://doi.org/10.1080/13825589508256588

[4] Fraser, S. and Bherer, L. (2013). Age-Related Decline in Divided-Attention: From Theoretical Lab Research to Practical Real-Life Situations. Wiley Interdisciplinary Reviews Cognitive Science, 4, 623-640. https://doi.org/10.1002/wcs.1252

[5] Knoblich, G. and Sebanz, N. (2006) The Social Nature of Perception and Action. Current Directions in Psychological Science, 15, 99-104.

https://doi.org/10.1111/j.0963-7214.2006.00415.x

[6] Gallese, V,. Fadiga, L., Fogassi, L. and Rizzolatti, G. (1996) Action Recognition in the Premotor Cortex. Brain, 119, 593-609. https://doi.org/10.1093/brain/119.2.593

[7] Rizzolatti, G., Fadiga, L., Gallese, V. and Fogassi, L. (1996) Premotor Cortex and the Recognition of Motor Actions. Cognitive Brain Research, 3, 131-141.

https://doi.org/10.1016/0926-6410(95)00038-0

[8] Atmaca, S., Sebanz, N. and Knoblich, G. (2011) The Joint Flanker Effect: Sharing Tasks with Real and Imagined Co-Actors. Experimental Brain Research, 211, 371-385. https://doi.org/10.1007/s00221-011-2709-9

[9] Atmaca, S., Sebanz, N., Prinz, W. and Knoblich G. (2008) Action Co-Representation: The Joint SNARC Effect. Social Neuroscience, 3, 410-420. https://doi.org/10.1080/17470910801900908

[10] Böckler, A., Knoblich, G. and Sebanz, N. (2012) Effects of a Coactor's Focus of Attention on Task Performance. Journal of Experimental Psychology, Human Perception and Performance, 38, 1404-1415. https://doi.org/10.1037/a0027523

[11] Sebanz, N., Knoblich, G. and Prinz, W. (2003) Representing Others' Actions: Just Like One's Own? Cognition, 88, B11-B21.

https://doi.org/10.1016/S0010-0277(03)00043-X

[12] Dolk, T., Hommel, B., Colzato, L.S., Schütz-Bosbach, S., Prinz, W. and Liepelt, R. (2014) The Joint Simon Effect: A Review and Theoretical Integration. Frontiers in Psychology, 5, 974. https://doi.org/10.3389/fpsyg.2014.00974

[13] Knoblich, G., Butterfill, S. and Sebanz, N. (2011) Psychological Research on Joint Action: Theory and Data. In: Ross, B., Ed., The Psychology of Learning and Motivation, Vol. 54, Academic Press, Burlington, 59-101. https://doi.org/10.1016/B978-0-12-385527-5.00003-6

[14] Baus, C., Sebanz, N., de la Fuente, V., Branzi, F.M., Martin, C. and Costa, A. (2014) On Predicting Others' Words: Electrophysiological Evidence of Prediction in Speech Production. Cognition, 133, 395-407. https://doi.org/10.1016/j.cognition.2014.07.006

[15] Kato, K., Yoshizaki, K. and Kimura, Y. (2016) Priority for One's Own Stimulus in Joint Performance: Evidence from an Event-Related Potential Study. NeuroReport, 27, 564-567. https://doi.org/10.1097/WNR.0000000000000566

[16] Sebanz, N., Knoblich, G., Prinz, W. and Wascher, E. (2006) Twin Peaks: An ERP Study of Action Planning and Control in Co-Acting Individuals. Journal of Cogni- 
tive Neuroscience, 18, 859-870. https://doi.org/10.1162/jocn.2006.18.5.859

[17] Tsai, C.C., Kuo, W.J., Hung, D.L. and Tzeng, O.J. (2008) Action Co-Representation Is Tuned to Other Humans. Journal of Cognitive Neuroscience, 20, 2015-2024. https://doi.org/10.1162/jocn.2008.20144

[18] Tsai, C.-C., Kuo, W.-J., Jing, J.-T., Hung, D.L. and Tzeng, O.J.-L. (2006) A Common Coding Framework in Self-Other Interaction: Evidence from Joint Action Task. Experimental Brain Research, 175, 353-362. https://doi.org/10.1007/s00221-006-0557-9

[19] Falkenstein, M., Koshlykova, N.A., Kiroj, V.N., Hoormann, J. and Hohnsbein, J. (1995) Late ERP Components in Visual and Auditory Go/Nogo Tasks. Electroencephalography and Clinical Neurophysiology, 96, 36-43. https://doi.org/10.1016/0013-4694(94)00182-K

[20] van Dinteren, R., Arns, M., Jongsma, M.L.A. and Kessels, R.P.C. (2014) P300 Development across the Life Span: A Systematic Review and Meta-Analysis. PloS One, 9, e87347. https://doi.org/10.1371/journal.pone.0087347

[21] Kok, A. (2001) On the Utility of P3 amplitude as a Measure of Processing Capacity. Psychophysiology, 38, 557-577.

[22] Polich, J. (2007) Updating P300: An Integrative Theory of P3a and P3b. Clinical Neurophysiology, 118, 2128-2148. https://doi.org/10.1016/j.clinph.2007.04.019

[23] Wronka, E., Kuniecki, M., Kaiser, J. and Coenen, A.M.L. (2007) The P3 Produced by Auditory Stimuli Presented in a Passive and Active Condition: Modulation by Visual Stimuli. Acta Neurobiologiae Experimentalis, 67, 155-164.

[24] Donchin, E. and Coles, M.G.H. (1988) Is the P300 Component a Manifestation of Context Updating? Behavioral and Brain Sciences, 11, 357-374. https://doi.org/10.1017/S0140525X00058027

[25] Dien, J., Spencer, K.M. and Donchin, E. (2004) Parsing the Late Positive Complex: Mental Chronometry and the ERP Components That Inhabit the Neighborhood of the P300. Psychophysiology, 41, 665-678.

[26] McCarthy, G. and Donchin, E. (1981) A Metric for Thought: A Comparison of P300 Latency and Reaction Time. Science, 211, 77-80.

https://doi.org/10.1126/science.7444452

[27] Williams, K.D., Cheung, C.K.T. and Choi, W. (2000) Cyberostracism: Effects of Being Ignored over the Internet. Journal of Personality and Social Psychology, 79, 748-762. https://doi.org/10.1037/0022-3514.79.5.748

[28] Katayama, J. and Polich, J. (1996) P300, Probability, and the Three-Tone Paradigm. Electroencephalography and Clinical Neurophysiology, 100, 555-562. https://doi.org/10.1016/S0168-5597(96)95171-0

[29] Katayama, J. and Polich, J. (1999) Auditory and Visual P300 Topography from a 3 Stimulus Paradigm. Clinical Neurophysiology, 110, 463-468. https://doi.org/10.1016/S1388-2457(98)00035-2

[30] Kaufman, D.A., Keith, C.M. and Perlstein, W.M. (2016) Orbitofrontal Cortex and the Early Processing of Visual Novelty in Healthy Aging. Frontiers in Aging Neuroscience, 8, 101. https://doi.org/10.3389/fnagi.2016.00101

[31] Folstein, M.F., Folstein S.E. and McHugh, P.R. (1975) "Minimental State": A Practical Method for Grading the Cognitive State of Patients for the Clinician. Journal of Psychiatric Research, 12, 189-198. https://doi.org/10.1016/0022-3956(75)90026-6

[32] Polich, J. (2003) Overview of P3a and P3b. In: Polich, J., Ed., Detection of Change: Event-Related Potential and fMRI Findings, Kluwer Academic Press, Boston, 83-98. 
https://doi.org/10.1007/978-1-4615-0294-4_5

[33] Cabeza, R., Anderson, N.D., Locantore, J.K. and McIntosh, A.R. (2002) Aging Gracefully: Compensatory Brain Activity in High-Performing Older Adults. Neuroimage, 17, 1394-1402. https://doi.org/10.1006/nimg.2002.1280

[34] Daffner, K.R., Sun, X., Tarbi, E.C., Rentz, D.M., Holcomb, P.J. and Riis, J.L. (2011) Does Compensatory Neural Activity Survive Old-Old Age? Neuroimage, 54, 427-438. https://doi.org/10.1016/j.neuroimage.2010.08.006 